\begin{tabular}{|c|l|}
\hline Title & A role of the Ca2+ binding site of DC-SIGN in the phagocytosis of E. coli \\
\hline Author(s) & Iyori, Mitsuhiro; Ohtani, Makoto; Hasebe, A kira; Totsuka, Y asunori; Shibata, Ken-ichiro \\
\hline Citation & Biochemical and biophysical research communications, 377 ( 2 ) , 367-372 \\
\hline Issue Date & 2008-10-10 \\
\hline Doc URL & http://hdl.handle.net/2115/43954 \\
\hline Type & article(author version) \\
\hline File Information & BBRC080925.pdf \\
\hline
\end{tabular}

Instructions for use 


\section{A role of the $\mathrm{Ca}^{2+}$ binding site of DC-SIGN in the phagocytosis of $E$. coli}

Mitsuhiro Iyori ${ }^{\text {a, }}{ }^{\text {c }}$, Makoto Ohtani ${ }^{\text {a, }}$, Akira Hasebe ${ }^{a}$, Yasunori Totsuka ${ }^{b}$ and Ken-ichiro Shibata ${ }^{a} *$

Divisions of Oral Molecular Microbiology ${ }^{a}$ and Oral and Maxillofacial Surgery ${ }^{b}$, Department of Oral Pathobiological Science, Hokkaido University Graduate School of Dental Medicine, Sapporo, Japan

${ }^{c}$ Research Fellow of the Japan Society for the Promotion of Science, Japan

*Corresponding author; Ken-ichiro Shibata

Division of Oral Molecular Microbiology, Department of Oral Pathobiological Science, Hokkaido University Graduate School of Dental Medicine, Nishi 7, Kita 13, Kita-ku, Sapporo 060-8586, Japan.

Phone: +81 11706 4240, FAX: +81 117064901

E-mail: shibaken@den.hokudai.ac.jp

Abbreviations: DCs, dendritic cells; ICAM-3, intracellular adhesion molecule-3; DC-SIGN, DC-specific ICAM-3-grabbing non-integrin; CRD, carbohydrate recognition domain; HEK, human embryonic kidney; TLRs, Toll-like receptors; NF-кB, nuclear factor- $\kappa \mathrm{B}$; ITAM, immunoreceptor tyrosine-based activation motif. 


\section{Abstract}

HEK293 cells stably expressing DC-SIGN (293/DC-SIGN) were examined for phagocytosis of Escherichia coli. 293/DC-SIGN stable transfectants were able to mediate phagocytosis of E. coli. The phagocytosis was inhibited by EDTA or several inhibitors specific for Syk kinase, Raf kinase and the transcription factor NF- $\kappa$ B. DC-SIGN consists of characteristic domains and motifs such as CRD, neck, incomplete ITAM, dileucine and tri-acidic cluster. HEK293 cells expressing mutants of DC-SIGN were also examined for the phagocytosis. It was found that $\mathrm{Ca}^{2+}$ binding sites in the CRD of DC-SIGN were involved in phagocytosis of bacteria as well as multimerization of DC-SIGN, and the neck region played a role in efficiency of binding to microbes as well as multimerization of the protein. 


\section{Introduction}

DCs express a wide variety of surface lectins that mediate detection of self and foreign carbohydrates [1-3]. Many of these lectins are members of the calcium-dependent C-type lectin family and have been associated with antigen uptake [1]. C-type lectins bind sugars through highly conserved CRDs [1]. These CRDs contain calcium-binding pockets that are essential for carbohydrate ligand binding [1]. DC-SIGN was first identified as a DC-specific C-type lectin that regulates the first contact between DC and resting T cells by binding to ICAM-3 [4]. Human DC-SIGN is a $44 \mathrm{kDa}$ type II transmembrane receptor displaying a CRD that is separated from the transmembrane region and cytoplasmic tail by a neck domain that consists of seven tandem repeat and one imcomplete tandem repeat [2]. DC-SIGN as well as several C-type lectin-like receptors participate in pathogen recognition and phagocytosis [2, 3]. Especially, DC-SIGN has been studied well and functions as a receptor for micro-organisms such as viruses, bacteria, fungi and several parasites [2, 3]. DC-SIGN efficiently captures human immunodeficiency virus type $I$ in the periphery and facilitates its transport to secondary lymphoid organs rich in $\mathrm{T}$ cells, to enhance 
infection in trans of these target cells [3]. Sindbis virus and hepatitis $\mathrm{C}$ virus can utilize DC-SIGN or L-SIGN as attachment receptors [2, 3]. It has been demonstrated that Mycobacterium tuberculosis evades the host defense system through the interaction with DC-SIGN [2, 3]. DC-SIGN promotes adherence and phagocytosis of a nonpathogenic E. coli K-12 strain by HeLa cells expressing human DC-SIGN [5]. Immature DCs are able to internalize Candida albicans in specific DC-SIGN-enriched vesicles [2, 3], and bind to Schistosoma mansoni soluble egg antigens [3]. DC-SIGN is a functionally relevant receptor for binding and internalization of Leishmania pifanoi amastigotes on DCs [3].

Thus, DC-SIGN functions as a receptor for various types of microbes, but the mechanisms underlying DC-SIGN-mediated phagocytosis are not yet defined clearly.

In this study, HEK293 cells expressing human DC-SIGN were established and examined for the phagocytic activity toward E. coli. It was found that Raf-1 kinase, Syk kinase and NF- $\mathrm{KB}$ are involved in the phagocytosis and $\mathrm{Ca}^{2+}$ binding sites in the CRD of DC-SIGN are responsible for both phagocytosis of E. coli and multimerization of the protein. 


\section{Materials and methods}

Reagents. A mouse monoclonal antibody (mAb) against DC-SIGN (MAB161) was purchased from R\&D Systems, Inc., (Minneapolis, MN); the DC-SIGN-specific blocking Ab (AZN-D1) from Beckman Coulter, Inc., (Fullerton, CA); a mouse mAb against human $\beta$-actin (AC-15) from Abcam (Stockholm, Sweden); EDTA, cytochalasin-D and wortmannin from Sigma-Aldrich (St Louis, MO); BAY11-7082 and GW5074 from BIOMOL International (Plymouth Meeting, PA); and piceatannol, the Syk inhibitor sulfonamide 31 and LY294002 from Calbiochem (La Jolla, CA).

All other reagents were purchased from commercial sources and were of analytical or reagent grade.

Cell culture and transfection. HEK293 cells and HEK293 cells stably expressing DC-SIGN (293/DC-SIGN) [6] were maintained in Dulbecco's modified Eagle's medium (Sigma) supplemented with $10 \%$ (vol/vol) heat-inactivated fetal bovine serum (Gibco BRL, Rockville, MD), 100 units/ml penicillin (Sigma) and $100 \mu \mathrm{g} / \mathrm{ml}$ streptomycin (Sigma). All transfections were carried out using METAFECTENE ${ }^{\mathrm{TM}}$ 
Transfection Reagent (Biontex Laboratories GmbH, München, Germany) according to the manufacturer's instructions.

Recombinant plasmids. pUNO-hDC-SIGN1a was purchased from InvivoGen (San Diego, CA). The DC-SIGN mutants including DC-SIGN ${ }^{\Delta \mathrm{E} 347-\mathrm{N} 365}$ (a deletion mutant of the CRD region of $\mathrm{Glu}^{347}$ to $\mathrm{Asn}^{365}$ ), DC-SIGN ${ }^{\Delta \mathrm{I77}-\mathrm{L} 206}$ (a deletion mutant of the neck region of $\mathrm{Ile}^{77}$ to $\mathrm{Leu}^{206}$ ), DC-SIGN ${ }^{\mathrm{Y} 31 \mathrm{~S}}$ (a point mutant of ITAM with a substitution of $\mathrm{Tyr}^{31}$ to Ser) and DC-SIGN ${ }^{\mathrm{LL} / \mathrm{AA}}$ [points mutant of dileucine (LL) motif with substitutions of Leu ${ }^{14}$ to Ala and of Leu ${ }^{15}$ to Ala] were generated by the QuikChange site-directed mutagenesis kits (Stratagene, La Jolla, CA) with pUNO-hDC-SIGN1a as the template. The following primers (and their reverse complements) were used for mutagenesis: DC-SIGN ${ }^{\mathrm{EE} 347-\mathrm{N} 365}, 5^{\prime}$-CAGTATTGGAACAGAGGAGACGACAAAT-

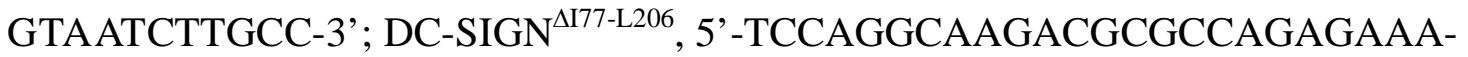
TCTAAG-3'; DC-SIGN ${ }^{\text {Y31S }}$, 5'-CAGACTCGAGGATCCAAGAGCTTAGCAGGG-3'; DC-SIGN ${ }^{\mathrm{LL} / \mathrm{AA}}, \quad$ 5'-CTGCAGCAGCTGGGCGCCGCGGAGGAGGAACAGCTG-3'. These mutations were confirmed by sequencing.

Phagocytosis. The phagocytosis was performed by the method described previously [6, 
7]. 293/DC-SIGN stable and transient transfectants $\left(2.5 \times 10^{5}\right)$ were seeded in each well of a 24 -well plate and then incubated at $37^{\circ} \mathrm{C}$ on the day before the assay. After the cells had been washed three times with base medium warmed at $37^{\circ} \mathrm{C}$, they were incubated for $1 \mathrm{~h}$ or $2 \mathrm{~h}$ with indicated doses of Alexa Fluor ${ }^{\circledR}$ 488-conjugated E. coli BioParticles $^{\circledR}$ (Invitrogen Co., Carlsbad, CA) or pHrodo ${ }^{\mathrm{TM}}$ E. coli BioParticles ${ }^{\circledR}$ (Invitrogen), which are nonfluorescent at neutral $\mathrm{pH}$, and bright red in acidic environments. After the cells had been washed three times with cold PBS, they were suspended in PBS containing 0.2\% (wt/vol) trypan blue to quench fluorescence caused by binding of bacteria to the surface of the cells and $1 \%(\mathrm{wt} / \mathrm{vol})$ paraformaldehyde to fix the cells. Flow cytometry was conducted using a FACS Calibur ${ }^{\circledR}$ machine (BD Biosciences, San Diego, CA) and CellQuest software (BD Biosciences). Phagocytosis was represented as two parameters; phagocytic activity and percentage of phagocytosis. Phagocytic activity was expressed as the mean fluorescence intensity (MFI) obtained by CellQuest software. Percentage of phagocytic cells was expressed as [number of cells taking up particles]/[total cell number analyzed (10000)] x 100. The phagocytosis assay by confocal laser scanning microscopy (CLSM) was previously described [6, 7]. 
Immunoblotting and cross-linking. Immunoblotting was performed as described using either an anti-DC-SIGN mAb (MAB161) or an anti- $\beta$-actin mAb as a primary $\mathrm{Ab}$ and an HRP-conjugated anti-mouse IgG (Jackson ImmunoResearch Laboratories, Inc., West Grove, PA) as a secondary $\mathrm{Ab}[6,7]$. Immunoreactive proteins were detected by using ECL $^{\mathrm{TM}}$ detection reagents (GE Healthcare, Piscataway, NJ). For cross-linking experiments, the cells were treated with a bis[sulfosuccinimidyl] suberate $\left(\mathrm{BS}^{3}\right.$, Pierce Biotechnology, Rockford, IL), a cell-surface protein crosslinker, at a final concentration of $5 \mathrm{mM}$ before lysed according to the manufacturer's instructions.

Surface expression of DC-SIGN in HEK293 transient transfectants. 293/DC-SIGN transient transfectants in each well of a 6-well plate were collected and were incubated at $4^{\circ} \mathrm{C}$ for $1 \mathrm{~h}$ with an anti-DC-SIGN mAb (MAB161) or an isotype-matched mouse IgG. The cells were then incubated with Alexa Fluor ${ }^{\circledR}$ 488-conjugated anti-mouse IgG Ab. The surface expression was measured using a FACS Calibur.

CLSM. 293/DC-SIGN transient transfectants were seeded on poly-L-lysine-coated coverslips in wells of a 6-well plate. The cells were fixed by methanol for 5 min at $-20^{\circ} \mathrm{C}$ and washed twice with PBS. The cells were incubated at room temperature for 
45 min with an anti-DC-SIGN mAb (MAB161) and then for another 45 min with an Alexa Fluor ${ }^{\circledR}$ 488-conjugated anti-mouse $\operatorname{IgG} \mathrm{Ab}$. The cells were washed three times with PBS and observed using an LSM410 invert Laser Scan Microscope (Carl Zeiss, Tokyo, Japan) using a 63 x objective (Leica Microsystems, Tokyo, Japan). 


\section{Results and discussion}

Phagocytosis of E. coli by HEK293 cells expressing DC-SIGN

To investigate roles of DC-SIGN in bacterial phagocytosis, 293/DC-SIGN stable transfectants were established and examined for phagocytosis of the bioparticles of Alexa Fluor ${ }^{\circledast}$ 488-E. coli. Phagocytosis was assayed under serum-free condition to exclude the involvement of Fc and/or complement receptors in the phagocytosis. The bioparticles were internalized by 293/DC-SIGN stable transfectants (Fig. 1A), but not by HEK293 cells (Fig. 1B). Then, HEK293 cells were transiently transfected with various amounts of the DC-SIGN construct and examined for phagocytosis of the bioparticles. It was found that 293/DC-SIGN transient transfectants internalized the bioparticles in a dose-dependent manner (Fig. 1C). Next experiment was carried out to determine whether the endosomes containing the bioparticles maturate to be acidic ones. For this purpose, pHrodo- $E$. coli bioparticles were used as bacterial targets, because they emit red fluorescence in the acidic environment. As shown in Fig. 1D, 293/DC-SIGN stable transfectants with internalized particles increased as the number of pHrodo-E. coli bioparticles added increased. This result suggests that $E$. coli bioparticles 
are internalized into 293/DC-SIGN stable transfectants and that the endosomes containing the bioparticles mature to be late endosome. In addition, it was found that the activity of 293/DC-SIGN stable transfectants to internalize E. coli bioparticles was significantly reduced by the addition of the DC-SIGN blocking Ab AZN-D1 at all ratios of cell to bacterium (Fig. 1E). Cytochalasin-D, an inhibitor of actin microfilaments rearrangement, reduced the internalization of $E$. coli bioparticles in a dose-dependent manner (Fig. 1F).

Taken together, these results strongly suggest that DC-SIGN expressed on the cell surface of 293/DC-SIGN stable transfectants is able to mediate phagocytosis of the gram-negative bacterium E. coli.

Molecular mechanisms underlying DC-SIGN-mediated phagocytosis of E. coli

To assess the mechanisms underlying the DC-SIGN-mediated phagocytosis of E. coli, we first examined for the effects of EDTA, a $\mathrm{Ca}^{2+}$ chelator, on the phagocytosis assay, because DC-SIGN classified into a C-type lectin family binds carbohydrate residues in a $\mathrm{Ca}^{2+}$-dependent manner [2]. It was found that $5 \mathrm{mM}$ EDTA markedly attenuated the 
percentage of phagocytosis from $73.2 \%$ to $18.4 \%$ (Fig. 2A) and the phagocytic activity (MFI) by $96 \%$ (Fig. 2B). This result suggests that the CRD of DC-SIGN is involved in the interaction with E. coli particles. Several lines of evidence on DC-SIGN-mediated signaling pathways have been accumulated [8-10]. DC-SIGN is colocalized with the protein-tyrosine kinases Syk and Lyn, Src-family kinases, in lipid raft-enriched microdomain, which are involved in immunoreceptor signaling in lymphoid and myeloid cells [9]. In addition, it has been demonstrated that ligation of DC-SIGN on human DCs induces activation of Raf-1, which subsequently induces acetylation of RelA (p65) of the transcription factor NF- $\kappa B$ only after TLR4-induced activation of $\mathrm{NF}-\kappa \mathrm{B}[8]$.

However, it still remains unknown whether these signaling molecules are involved in the DC-SIGN-mediated phagocytosis. Therefore, next experiment was carried out to determine whether inhibitors specific for these signaling proteins affect the DC-SIGN-mediated phagocytosis. The NF-кB inhibitor BAY11-7082 markedly attenuated the phagocytic activity of 293/DC-SIGN stable transfectants (Fig. 2C), suggesting that NF- $\kappa$ B plays an important role in the DC-SIGN-mediated phagocytosis. 
This is consistent with our previous finding that TLR-mediated signals leading to NF- $\mathrm{B}$ activation upregulate phagocytosis of bacteria by macrophages [6]. The Raf-1 kinase inhibitor GW5074 remarkably reduced the DC-SIGN-mediated phagocytosis of E. coli in a dose-dependent manner (Fig. 2C). The Syk kinase inhibitor piceatannol also reduced the phagocytosis and, to a lesser extent, the Syk inhibitor sulfonamide 31 suppressed the phagocytosis (Fig. 2C). These data suggest that Syk and Raf-1 kinases are involved in bacterial phagocytosis mediated by DC-SIGN. Although the phosphatidylinositol 3-kinase (PI3K) inhibitor LY294002 inhibited the DC-SIGN-mediated phagocytosis, another PI3K inhibitor wortmannin did not (Fig. 2C). In addition, the Akt inhibitor $\mathrm{V}$ did not inhibit the phagocytosis (Fig. 2C). These results suggest that the PI3K/AKT pathway is not involved in the DC-SIGN-mediated phagocytosis. The MEK inhibitors U0126 and PD98059, and the p38 inhibitor SB203580 had no effect on the DC-SIGN-mediated phagocytosis (Fig. 2C), suggesting that the MAPK pathway is not involved in the DC-SIGN-mediated phagocytosis. This is not in agreement with our previous finding that the p38 inhibitor SB203580 reduced the bacterial phagocytosis by a monocytic cell line, THP-1 cells [7]. This disagreement 
suggests that the bacterial phagocytosis by professional phagocytes like THP-1 cells [7] is different from that by nonphagocytic cells transfected with the phagocytic receptor DC-SIGN and/or is not mediated by DC-SIGN alone. This speculation is supported by the findings that the PI3K inhibitor wortmannin did not inhibit the DC-SIGN-mediated phagocytosis (Fig. 2C), but did the phagocytosis by macrophages [7].

Taken together, these results suggest that $\mathrm{Ca}^{2+}$ ions are required for the DC-SIGN-mediated phagocytosis of E. coli, which is regulated by Syk and Raf kinases as well as the transcription factor NF- $\mathrm{KB}$. In addition, it has been demonstrated that the Syk inhibitor piceatannol did not inhibit phagocytosis of zymosan by HEK293 cells expressing Dectin-1 [11]. Therefore, it is speculated that the DC-SIGN-mediated bacterial phagocytosis uses the signaling pathway different from the phagocytosis by Dectin-1, although both DC-SIGN and Dectin-1 are members of the C-type lectin superfamily and have functional and structural similarity $[2,12]$.

Contribution of $\mathrm{Ca}^{2+}$ binding site of DC-SIGN to the phagocytosis of E. coli

It has been demonstrated that DC-SIGN consists of characteristic domains and motifs 
such as CRD, neck, incomplete ITAM, dileucine (LL) and tri-acidic (EEE) cluster [2]. The CRD of DC-SIGN consisting of four amino acids (Glu347, Asn349, Glu354 and Asn365) interacts with $\mathrm{Ca}^{2+}$ and are involved in the recognition of specific carbohydrate structures [2]. The CRD of DC-SIGN is separated from the transmembrane (TM) regions by a neck domain that consists of seven complete and one imcomplete tandem repeat (Fig. 3A). The neck domain mediates multimerization of DC-SIGN, which is required for binding of ligands or pathogens $[13,14]$. The cytoplasmic tail of DC-SIGN includes internalization motifs, such as the LL and the EEE cluster [2, 15], and an incomplete ITAM which is considered to be involved in signal transduction pathway [2, $15]$.

In order to determine which motifs in DC-SIGN play a key role in bacterial phagocytosis, we made several mutants of DC-SIGN (Fig. 3A) and were transfected into HEK293 cells. It was found that DC-SIGN ${ }^{\mathrm{Y} 31 \mathrm{~S}}$ could mediate the phagocytosis of $E$. coli, but DC-SIGN ${ }^{\Delta I 77-\mathrm{L} 206}$ and $\mathrm{DC}-S I G N^{\mathrm{LL} / \mathrm{AA}}$ reduced the phagocytosis, and DC-SIGN ${ }^{\Delta \mathrm{E} 347-\mathrm{N} 365}$ did not (Fig. 3B). We thought that these differences in the activities of the four mutants might be attributed to the differences in the surface expression 
levels and/or the conformation of the binding site of DC-SIGN. To gain insights into mechanisms responsible for these differences in the phagocytosis, we examined whether these mutants of DC-SIGN are expressed on the surface of HEK293 transfectants. DC-SIGN $^{\text {WT }}$ and mutants were expressed in HEK293 cells (Fig. 4A). Flow cytometric analysis showed that the mutants were expressed on the cell surfaces of their transfectants, although their expression levels varied (Fig. 4B). Surprisingly, even $\mathrm{DC}-S I G N^{\Delta \mathrm{I77-L206}}$ lacking the neck region responsible for oligomerization was expressed on the surfaces, although the expression level was significantly reduced in comparison with that of DC-SIGN ${ }^{\mathrm{WT}}$ (Fig. 4B). This result suggests that these mutations, except for $\mathrm{DC}-\mathrm{SIGN}^{\mathrm{AE} 347-\mathrm{N} 365}$, do not have effects that are strong enough to abrogate the expression of DC-SIGN on the cell surface. However, DC-SIGN ${ }^{\Delta \mathrm{E} 347-\mathrm{N} 365}$ lacking CRD region responsible for the association with $\mathrm{Ca}^{2+}$ was not expressed on the cell surface (Fig. 4B). In addition, CLSM showed that the DC-SIGN ${ }^{\triangle \mathrm{E} 347-\mathrm{N} 365}$ protein was localized only in cytosol, but proteins of other mutants were localized specifically in the cell membrane (Fig. 4C). These results suggest that $\mathrm{Ca}^{2+}$ binding site in the CRD plays important roles in the surface expression as well as the interaction with $\mathrm{Ca}^{2+}$ and 
microbes. It has been demonstrated that the neck domain of murine DC-SIGN responsible for tetramer formation is involved in efficient binding to multivalent ligands [16] and the impairment in tetramer formation results in the impairment of the binding to pathogens/ligands [14]. However, the involvement of $\mathrm{Ca}^{2+}$ binding site of DC-SIGN in its multimerization still remains unclear. Therefore, next experiment was carried out to determine the oligomeric states of the mutants by using a chemical cross-linking reagent $\mathrm{BS}^{3}$. It was found that in the presence of $\mathrm{BS}^{3}$ several bands corresponding to tetramer and monomer were detected in the transfectants of DC-SIGN ${ }^{\mathrm{WT}}$ as well as DC-SIGN $^{\mathrm{Y} 31 \mathrm{~S}}$ (lanes 7 and 10 in Fig. 4D); only monomer band in DC-SIGN ${ }^{\Delta \mathrm{E} 347-\mathrm{N} 365}$ (lane 8 in Fig. 4D); bands corresponding to dimers, trimers and tetramers in

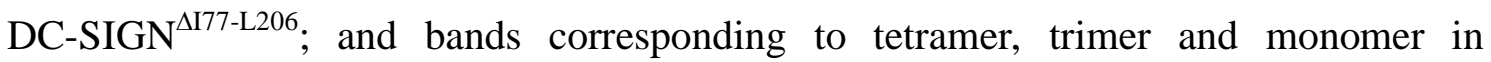
DC-SIGN $^{\text {LL/AA }}$ (lane 11 in Fig. 4D). These results suggest that the extent of multimerization is well correlated to the phagocytic activity, and also suggest that the $\mathrm{Ca}^{2+}$ binding site in the CRD and the region of I77 to L206 in the neck domain of DC-SIGN is required for the tetramer formation. Treatment of monocyte-derived DCs with H-200, an Ab against amino acids 61-200 in the neck domain of DC-SIGN, 
displayed profile of phosphotyrosine- and phosphoserine-containing proteins distinct from that with ICAM-3 and other Abs against DC-SIGN, including 120507, C20 and IB10 [10]. Moreover, the signaling pathway triggered by the Ab $\mathrm{H}-200$ is similar to that by human immunodeficiency virus 1 infection [10]. The present study demonstrated that neck region of DC-SIGN plays roles in efficiency of binding to microbes as well as multimerization of the protein. This study also demonstrated that $\mathrm{Ca}^{2+}$ binding site in the CRD of DC-SIGN are involved in phagocytosis of bacteria as well as multimerization of DC-SIGN.

Taken together, it is considered that multimerization, possibly tetramerization, of DC-SIGN is required for phagocytosis of bacteria.

\section{Acknowledgments}

This work was supported by Grants-in-Aid for Scientific Research B 19390477 provided by the Japan Society for the Promotion of Science (JSPS). One of the authors

(M. I.) appreciates the JSPS Research Fellowships for Young Scientists. 


\section{References}

[1] C.G. Figdor, Y. van Kooyk, G.J. Adema, C-type lectin receptors on dendritic cells and Langerhans cells, Nat Rev Immunol 2 (2002) 77-84.

[2] Y. van Kooyk, T.B. Geijtenbeek, DC-SIGN: escape mechanism for pathogens, Nat Rev Immunol 3 (2003) 697-709.

[3] A. Cambi, M. Koopman, C.G. Figdor, How C-type lectins detect pathogens, Cell Microbiol 7 (2005) 481-488.

[4] T.B. Geijtenbeek, R. Torensma, S.J. van Vliet, G.C. van Duijnhoven, G.J. Adema, Y. van Kooyk, C.G. Figdor, Identification of DC-SIGN, a novel dendritic cell-specific ICAM-3 receptor that supports primary immune responses, Cell 100 (2000) 575-585.

[5] P. Zhang, S. Snyder, P. Feng, P. Azadi, S. Zhang, S. Bulgheresi, K.E. Sanderson, J. He, J. Klena, T. Chen, Role of N-acetylglucosamine within core lipopolysaccharide of several species of gram-negative bacteria in targeting the DC-SIGN (CD209), J Immunol 177 (2006) 4002-4011.

[6] M. Iyori, H. Kataoka, H.M. Shamsul, K. Kiura, M. Yasuda, T. Nakata, A. Hasebe, K. Shibata, Resveratrol Modulates Phagocytosis of Bacteria through an NF-кB-Dependent 
Gene Program, Antimicrob Agents Chemother 52 (2008) 121-127.

[7] M. Mae, M. Iyori, M. Yasuda, H.M. Shamsul, H. Kataoka, K. Kiura, A. Hasebe, Y. Totsuka, K.I. Shibata, The diacylated lipopeptide FSL-1 enhances phagocytosis of bacteria by macrophages through a Toll-like receptor 2-mediated signalling pathway, FEMS Immunol Med Microbiol 49 (2007) 398-409.

[8] S.I. Gringhuis, J. den Dunnen, M. Litjens, B. van Het Hof, Y. van Kooyk, T.B. Geijtenbeek, C-type lectin DC-SIGN modulates Toll-like receptor signaling via Raf-1 kinase-dependent acetylation of transcription factor NF-אB, Immunity 26 (2007) 605-616.

[9] E. Caparros, P. Munoz, E. Sierra-Filardi, D. Serrano-Gomez, A. Puig-Kroger, J.L. Rodriguez-Fernandez, M. Mellado, J. Sancho, M. Zubiaur, A.L. Corbi, DC-SIGN ligation on dendritic cells results in ERK and PI3K activation and modulates cytokine production, Blood 107 (2006) 3950-3958.

[10] A. Hodges, K. Sharrocks, M. Edelmann, D. Baban, A. Moris, O. Schwartz, H. Drakesmith, K. Davies, B. Kessler, A. McMichael, A. Simmons, Activation of the lectin DC-SIGN induces an immature dendritic cell phenotype triggering Rho-GTPase activity 
required for HIV-1 replication, Nat Immunol 8 (2007) 569-577.

[11] D.M. Underhill, E. Rossnagle, C.A. Lowell, R.M. Simmons, Dectin-1 activates Syk tyrosine kinase in a dynamic subset of macrophages for reactive oxygen production, Blood 106 (2005) 2543-2550.

[12] G.D. Brown, Dectin-1: a signalling non-TLR pattern-recognition receptor, Nat Rev Immunol 6 (2006) 33-43.

[13] H. Feinberg, D.A. Mitchell, K. Drickamer, W.I. Weis, Structural basis for selective recognition of oligosaccharides by DC-SIGN and DC-SIGNR, Science 294 (2001) 2163-2166.

[14] D. Serrano-Gomez, E. Sierra-Filardi, R.T. Martinez-Nunez, E. Caparros, R. Delgado, M.A. Munoz-Fernandez, M.A. Abad, J. Jimenez-Barbero, M. Leal, A.L. Corbi, Structural requirements for multimerization of the pathogen receptor dendritic cell-specific ICAM3-grabbing non-integrin (CD209) on the cell surface, J Biol Chem 283 (2008) 3889-3903.

[15] A. Engering, T.B. Geijtenbeek, Y. van Kooyk, Immune escape through C-type lectins on dendritic cells, Trends Immunol 23 (2002) 480-485. 
[16] T. Gramberg, T. Zhu, C. Chaipan, A. Marzi, H. Liu, A. Wegele, T. Andrus, H. Hofmann, S. Pohlmann, Impact of polymorphisms in the DC-SIGNR neck domain on the interaction with pathogens, Virology 347 (2006) 354-363. 


\section{Figure legends}

Figure 1. Phagocytosis of $E$. coli by HEK293 cells expressing DC-SIGN. 293/DC-SIGN stable transfectants (A) or HEK293 cells (B) were incubated for $1 \mathrm{~h}$ with Alexa Fluor $^{\circledR} 488$-conjugated E. coli at a cell:bacterium ratio of 1:5. Cells were stained with Alexa Fluor ${ }^{\circledR}$ 594-conjugated concanavalin A, and then fixed and imaged by CLSM. (C) HEK293 transient transfectants, which were established by being $24 \mathrm{~h}$ with various concentrations of a pUNO-DC-SIGN plasmid, were examined for $1 \mathrm{~h}$ with

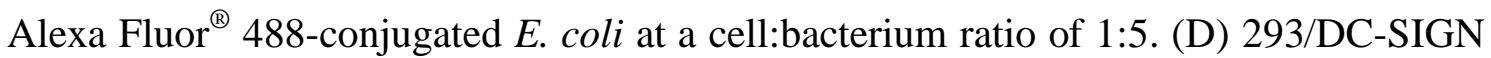
stable transfectants were incubated for $2 \mathrm{~h}$ with various concentrations of pHrodo ${ }^{\circledR}$-conjugated E. coli. (E) 293/DC-SIGN stable transfectants were treated for $1 \mathrm{~h}$ with $10 \mu \mathrm{g} / \mathrm{ml}$ of the DC-SIGN-blocking Ab AZN-D1, and then incubated for $1 \mathrm{~h}$ with Alexa Fluor ${ }^{\circledR}$ 488-conjugated E. coli. (F) 293/DC-SIGN stable transfectants were treated for $1 \mathrm{~h}$ with cytochalasin $\mathrm{D}$, and then incubated for $1 \mathrm{~h}$ with Alexa Fluor ${ }^{\circledR}$ 488-conjugated E. coli. Results are representatives of three independent experiments.

Figure 2. Effects of several chemicals on DC-SIGN-mediated phagocytosis of $E$. 
coli. 293/DC-SIGN stable transfectants were treated for $1 \mathrm{~h}$ with or without $5 \mathrm{mM}$ EDTA, and then examined for another $1 \mathrm{~h}$ with Alexa Fluor ${ }^{\circledR} 488$-conjugated E. coli at a cell:bacterium ratio of $1: 10$. The phagocytosis was expressed by the percentage of phagocytosis (A) and the phagocytic activity (B). (C) 293/DC-SIGN stable transfectants were treated for $1 \mathrm{~h}$ with indicated doses of chemicals: GW5074, a Raf-1 inhibitor; BAY11-7082, a NF-кB inhibitor; piceatannol and sulfonamide 31, a Syk inhibitors; LY294002 and wortmannin; a PI3K inhibitors; Akt inhibitor V, a Akt inhibitor; U0126 and PD98059, a MEK inhibitors; and SB203580, a p38 MAPK inhibitor. The cells were then incubated for $1 \mathrm{~h}$ with Alexa Fluor ${ }^{\circledR}$ 488-conjugated E. coli at a cell:bacterium ratio of $1: 10$. Phagocytosis is expressed by relative activity, where the activity without inhibitors is taken as $100 \%$. Results are representatives of three independent experiments.

Figure 3. Phagocytosis of $E$. coli by HEK293 cells expressing DC-SIGN mutants.

(A) Deletion and point mutants of human DC-SIGN are schematically shown: WT, a wild type DC-SIGN; $\triangle \mathrm{E} 347-\mathrm{N} 365$, a deletion mutant of a $\mathrm{Ca}^{2+}$ binding site in the CRD; 
$\Delta$ I77-L206, a deletion mutant of the neck domain; Y31S, a point mutant of the cytoplasmic hemi-ITAM; and LL/AA, a double point mutant of the di-leucine motif. Symbols: TM, transmembrane domain; CRD, carbohydrate-recognition domain; and Neck, neck domain. The amino acid is written as a single-letter code in the cytoplasmic domain of DC-SIGN. (B) HEK293 cells were transiently transfected for 24h with 1000 ng of DC-SIGN ${ }^{\mathrm{WT}}$ or its mutants. The cells were then incubated for $1 \mathrm{~h}$ with Alexa Fluor $^{\circledR}$ 488-conjugated $E$. coli at a cell:bacterium ratio of 1:10. The phagocytosis was expressed by percentage of phagocytosis. Results are representatives of five independent experiments.

Figure 4. Expression profiles of DC-SIGN mutants. (A) HEK293 cells were transfected by being incubated for $24 \mathrm{~h}$ with DC-SIGN ${ }^{\mathrm{WT}}$ (Lane 2), DC-SIGN ${ }^{\Delta \mathrm{E} 347-\mathrm{N} 365}$ (Lane 3), DC-SIGN ${ }^{\Delta 177-\mathrm{L} 206}$ (Lane 4), DC-SIGN ${ }^{\mathrm{Y} 31 \mathrm{~S}}$ (Lane 5), DC-SIGN ${ }^{\mathrm{LL} / \mathrm{AA}}$ (Lane 6), or pUNO-empty (Lane 1). Cell lysates were separated on a 10\% gel and immunoblotted with a mAb against DC-SIGN (MAB161). (B) HEK293 transient transfectants were stained with MAB161 or isotype-matched mouse IgG. Ratios of MFI of the cells stained 
with MAB161 to that of a control are shown in each histogram. (C) HEK293 transient transfectants were stained with MAB161 and then with Alexa Fluor ${ }^{\circledR}$ 488-conjugated anti-mouse IgG Ab. The cells were fixed and imaged using the Zeiss 410 confocal microscope. (D) HEK293 cells were transiently transfected with DC-SIGN ${ }^{\text {WT }}$ (Lanes 2 and 7), DC-SIGN ${ }^{\Delta \mathrm{E} 347-\mathrm{N} 365}$ (Lanes 3 and 8), DC-SIGN ${ }^{\Delta \mathrm{I77-L206}}$ (Lanes 4 and 9), DC-SIGN $^{\text {Y31S }}$ (Lanes 5 and 10), DC-SIGN ${ }^{\text {LL/AA }}$ (Lanes 6 and 11), or pUNO-empty (Lane 1). The transfectants were treated with (Lanes 7-11) or without (Lanes 1-6) cross-linker $\mathrm{BS}^{3}$ at $5 \mathrm{mM}$. Cell lysates were separated on a $7.5 \%$ gel and immunoblotted with MAB161. Results are representatives of three independent experiments. 


\section{$25 \mu \mathrm{m}$}

\section{E. coli}

E. coli

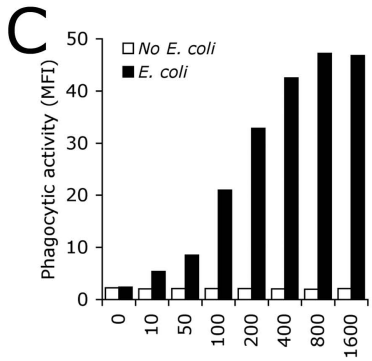

pUNO-DC-SIGN (ng/well)
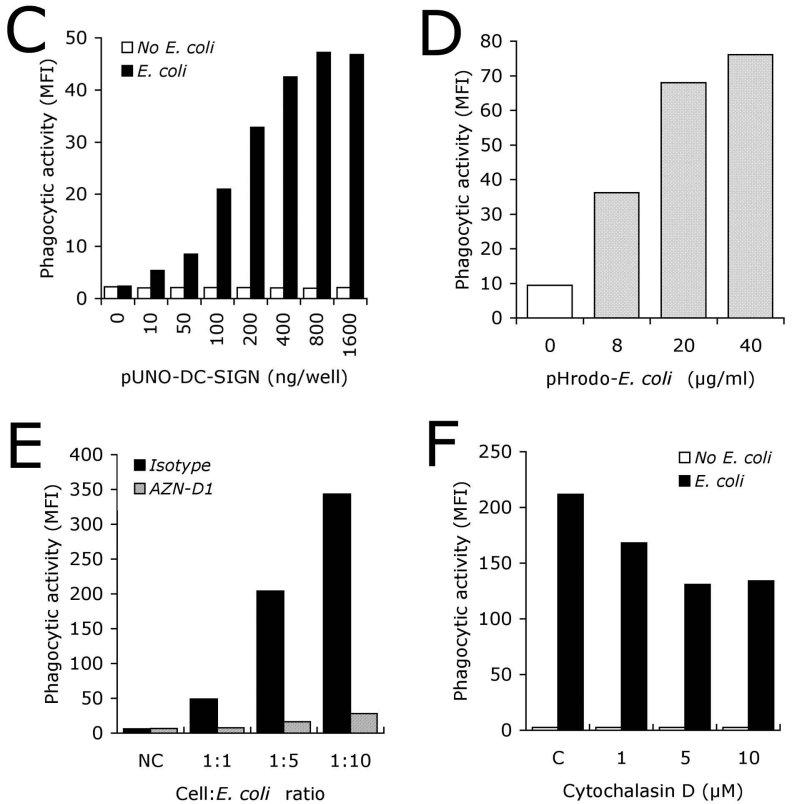


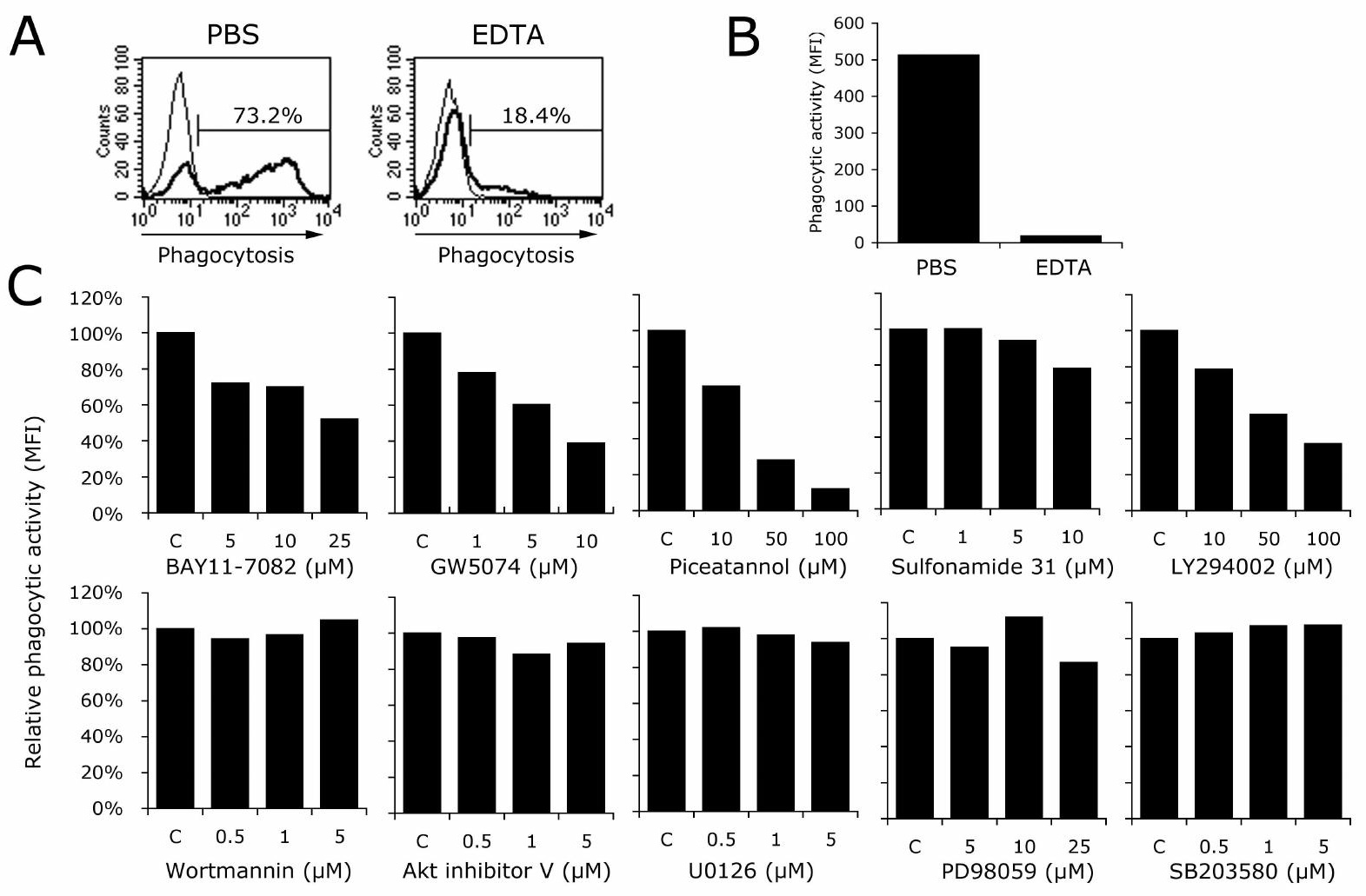




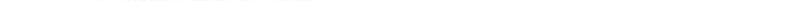


$\begin{array}{lllllll}1 & 2 & 3 & 4 & 5 & 6 & \text { B }\end{array}$

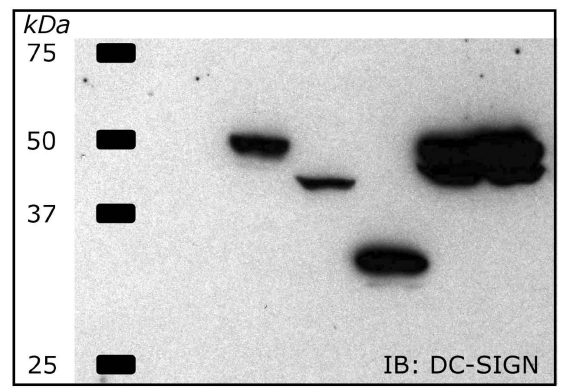

IB:
$\beta$-actin
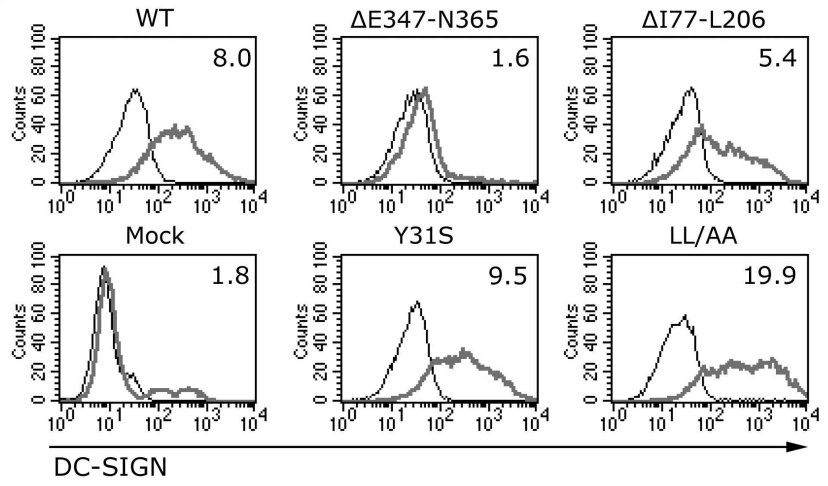

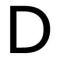

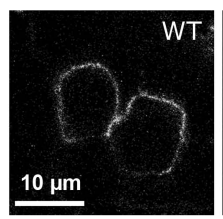

$\Delta E 347-N 365$

$x^{24}$

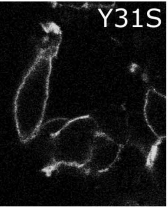

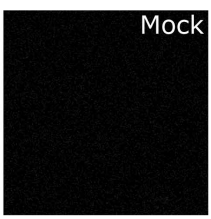

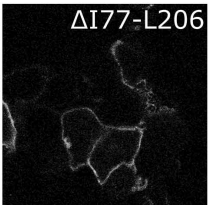

LL/AA

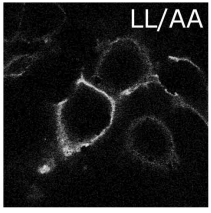

$\begin{array}{lllllllllll}1 & 2 & 3 & 4 & 5 & 6 & 7 & 8 & 9 & 10 & 11\end{array}$

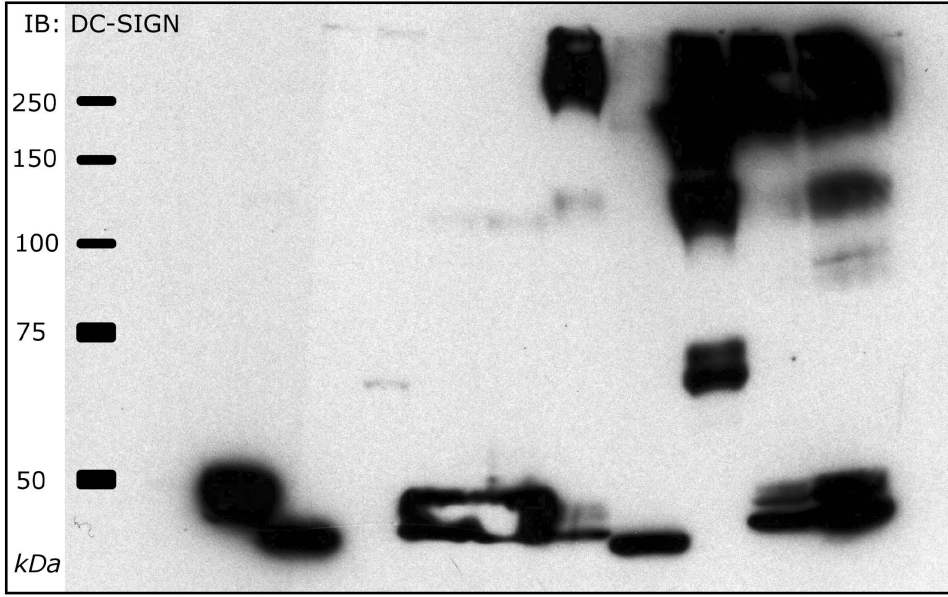

IB:

$\beta$-actin 